\title{
OPEN Effect of ball collision direction on a wet mechanochemical reaction
}

\author{
Takahiro Kozawa ${ }^{1 凶}$, Kayo Fukuyama ${ }^{1}$, Kizuku Kushimoto² ${ }^{2}$ Shingo Ishihara ${ }^{2}$, Junya Kano², \\ Akira Kondo ${ }^{1} \&$ Makio Naito ${ }^{1}$
}

Mechanochemical reactions can be induced in a solution by the collision of balls to produce high-temperature and high-pressure zones, with the reactions occurring through a dissolutionprecipitation mechanism due to a change in solubility. However, only a fraction of the impact energy contributes to the mechanochemical reactions, while the rest is mainly consumed by the wear of balls and the heat generation. To clarify whether the normal or tangential component of collisions makes a larger contribution on the reaction, herein we studied the effect of collision direction on a wet mechanochemical reaction through combined analysis of the experimental reaction rates and simulated ball motion. Collisions of balls in the normal direction were found to contribute strongly to the wet mechanochemical reaction. These results could be used to improve the synthesis efficiency, predict the reaction, and lower the wear in the wet mechanochemical reactions.

Mechanochemical reactions of solid phases using grinding equipment are a facile, green, and scalable synthetic approach for organic ${ }^{1,2}$, inorganic ${ }^{3}$, metallic ${ }^{4-6}$, and energy fields ${ }^{7,8}$. When solid reactants are milled, they experience mechanical stress and strain from collisions and shears with the milling body, which induce solid-solid reactions including interfacial dislocations and ion diffusion ${ }^{9-11}$. Another driving force of these non-equilibrium reaction is local frictional heat at the solid surface. However, such heat could also decompose thermodynamically unstable hydrous materials including hydrates and hydroxides ${ }^{12}$. Besides, the control of particle shapes is very difficult while applying external forces. An alternative is wet mechanochemical reactions based on a dissolution-precipitation mechanism instead of solid-solid reactions ${ }^{13-16}$. By utilizing the solution-based reaction mechanism, we were able to control the particle shape in synthesized hydrates and hydroxides despite the use of planetary ball milling ${ }^{17,18}$. However, to improve the versatility of this method for synthesizing functional particles, it is necessary to determine how the input mechanical energy acts on the solution or reactants.

Chemical interaction in the mechanochemical process may be modelled by collision theory due to its statistical nature ${ }^{19}$. The reaction rate $(v)$ in a mill can be estimated as

$$
v=K x S
$$

where $K$ is a constant characterizing the reaction probability at a given mechanical action per unit contact, $x$ is the probability for the particles to collide with a milling body per time, and $S$ is the contact area of the different reactants during mechanical action. In solution, the dissolution and dispersion of reactants reduce the contact probability between them as compared to that with the milling body (such as ball media). Consequently, the rate of wet mechanochemical reaction mainly depends on $K$, which is the impact energy in the collision between balls. Such collision causes high-temperature and high-pressure local zones within the solution ${ }^{20-22}$. This limited reaction field affects the local solubility of reactants and products, which in turn can induce the dissolution, nucleation, and crystal growth of solid species. Mechanically, the collision of balls can be divided into normal and tangential components. The impact energy $(E)$ is the sum of the two components:

$$
E=e_{n}+e_{t}
$$

where $e_{n}$ and $e_{t}$ are the impact energies in the normal and tangential directions, respectively. The grinding efficiency, defined as the energy to create a new surface for the energy input, is $\sim 15 \%$ at best for the comminution of quartz and soda-lime glass ${ }^{23}$, while most of the input mechanical energy does not contribute to the reaction. Therefore, determining which collision direction contributes the most to a wet mechanochemical reaction can

\footnotetext{
${ }^{1}$ Joining and Welding Research Institute, Osaka University, 11-1 Mihogaoka, Ibaraki, Osaka 567-0047, Japan. ${ }^{2}$ Institute of Multidisciplinary Research for Advanced Materials, Tohoku University, 2-1-1 Katahira, Aoba-ku, Sendai, Miyagi 980-8577, Japan. ${ }^{\circledR}$ email: t-kozawa@jwri.osaka-u.ac.jp
} 
help estimate the reaction rate from the input mechanical energy, and predict the most efficient milling conditions for producing the desired particles. There has been no discussion of particle synthesis by wet mechanochemical reactions from the ball collision direction.

In this study, we examined how the ball collision direction affects a wet mechanochemical reaction by combining experimentally measured formation rates with simulated ball motion in solution. The model reaction was the formation of lithium titanate hydrate $\left(\mathrm{Li}_{1.81} \mathrm{H}_{0.19} \mathrm{Ti}_{2} \mathrm{O}_{5} \cdot x \mathrm{H}_{2} \mathrm{O}\right.$, LHTO) through planetary ball milling of $\mathrm{LiOH}$ and $\mathrm{TiO}_{2}$ in water. The formation fraction of LHTO was measured as the treatment conditions were varied systematically. Meanwhile, our group had developed accessible simulation software (KIK DEM, ver.1.1) to reveal the motion of balls during wet milling, using the distinct element method (DEM) to treat the ball media and simplified modeling of interactions with fluids ${ }^{24,25}$. This software can output the collision frequency and the impact energies for each collision component. After fitting the formation fraction of LHTO and the calculated cumulative impact energy in each milling condition, the formation rate was found to follow the impact energy of the normal component, which increased linearly with the size and input of the balls. The values of the tangential component varied a great deal depending on the motion of balls in the milling vessel. However, the excess tangential impact energy was consumed without forming LHTO. The resulting guideline for wet mechanochemical reactions provides a highly efficient route for synthesizing thermodynamically unstable materials at room temperature.

\section{Results and discussion}

Many researchers have reported that LHTO is an attractive precursor of $\mathrm{Li}_{4} \mathrm{Ti}_{5} \mathrm{O}_{12}$ anode for $\mathrm{Li}$-ion batteries, due to its superior properties for constructing various morphologies ${ }^{26-28}$. However, the conventional hydrothermal and solvothermal synthesis routes for LHTO suffer from certain technical drawbacks, including the need of highly reactive titanium sources, restricted temperature-pressure conditions, and prolonged treatment time for nucleation and growth ${ }^{26-29}$. In comparison, in the mechanochemical reaction, LHTO is formed from inexpensive $\mathrm{TiO}_{2}$ by using high-energy grinding at room temperature to induce its dissolution in the solution ${ }^{17}$. The normally insoluble $\mathrm{TiO}_{2}$ can gradually dissolve in a strongly basic $\mathrm{LiOH}$ solution ${ }^{30,31}$. The collisions of balls trigger the formation of LHTO in the solution, while the formation rate depends on the ball size. When relatively small balls $(\phi 1 \mathrm{~mm})$ are used, the formation fraction of LHTO ( $\alpha$, estimated by the X-ray diffraction (XRD) peak ratios of $\mathrm{TiO}_{2}$ and LHTO) increases rapidly with an increase in the centrifugal acceleration and milling time (Fig. 1a). The centrifugal acceleration $(G)$ was calculated using technical data as revolution diameter and frequency of the motor and controlled by the revolution speed. The obtained result agrees with previous work using $\$ 2 \mathrm{~mm}$ balls ${ }^{17}$. In contrast, the formation rate is lower for larger balls of $\phi 5 \mathrm{~mm}$ (Fig. 1b). For example, under the milling condition of $150 \mathrm{G}$ and $3 \mathrm{~h}$, the value of $\alpha$ is $\sim 0.8$ for $\phi 1 \mathrm{~mm}$ balls but only 0.1 for $\phi 5 \mathrm{~mm}$ balls. When fixing the ball size at $\phi 1 \mathrm{~mm}$, a grows with time more slowly at low centrifugal accelerations. These results suggest that smaller balls improve the efficiency of LHTO formation.

The collision frequency per unit volume clearly depends on the ball size. Furthermore, an increase in ball input (i.e., more balls) also increases the collision frequency. The enhanced contact probabilities lead to more opportunity to form LHTO. Indeed, the a value increases with decreasing ball size and increasing ball input (Fig. 1c). As mentioned above, the formation rate is slow with larger balls when the other conditions are fixed. Under the milling condition of $200 \mathrm{~g}$ balls, $100 \mathrm{G}$, and $5 \mathrm{~h}$, the $\alpha$ value reaches 0.9 for $\phi 1 \mathrm{~mm}$ and $\phi 2 \mathrm{~mm}$ balls but remains at 0.45 for $\phi 5 \mathrm{~mm}$ balls. Although increasing the ball input accelerates the wet mechanochemical reaction, it also causes the generation of impurity due to wear. After milling with $50 \mathrm{~g}$ of $\$ 2 \mathrm{~mm}$ balls, the product ( $\alpha=0.4$ ) contained only LHTO and $\mathrm{TiO}_{2}$ (Fig. 1d). In contrast, a similar fraction of product could be formed using $200 \mathrm{~g}$ of $\phi 5 \mathrm{~mm}$ balls, but the product also exhibited diffraction peaks of $\mathrm{ZrO}_{2}$ as an impurity. The generation of impurity is unavoidable even for small balls if their input is high.

Overall, the experimentally measured formation of LHTO through wet mechanochemical reaction revealed the following findings when the other reaction parameters are fixed in each case. (1) The product is formed faster when using smaller balls. (2) The formation fraction increases with an increase in the centrifugal acceleration and treatment time. (3) Increasing the input of balls promotes the reaction. (4) A larger amount of balls (regardless of their size) induces the generation of impurity due to wear. Based on these, we hypothesize that the collision frequency of balls during milling controls the formation rate of LHTO. Next, this hypothesis was examined using simulation, which assumed that only the balls and water were present in the ball mill.

Firstly, we simulated the effects of centrifugal acceleration and ball size while fixing the ball input at $100 \mathrm{~g}$ (Fig. 2a). To reduce the computational load, the lower limit of ball size was set at $3 \mathrm{~mm}$. With increasing ball size, the impact energy per collision increases whereas the collision frequency decreases. The distribution width of the impact energy is about three orders of magnitude larger for smaller balls. Increasing the centrifugal acceleration from 50 to $150 \mathrm{G}$ leads to a shift of frequency distribution to higher regions, however the effect is less than that from changing the ball size. Next, we investigated the effect of ball input on the impact energy (Fig. 2b). The total impact energy $(E)$ is an integrated value of the impact energy per collision and the frequency. $E$ initially decreases with an increase in the ball input, and then it increases at above 100-140 g. However, the use of $\phi 8 \mathrm{~mm}$ balls leads to a rapid rise of impact energy with the input up to $80 \mathrm{~g}$, and then it decreases to a minimum value at $140 \mathrm{~g}$. When an excess volume of balls is used, the impact energy is higher with smaller balls in Fig. $2 \mathrm{~b}$. The same trend was observed under lower centrifugal accelerations of $50 \mathrm{G}$ and $100 \mathrm{G}$ (Fig. S1).

To clarify the dependency of impact energy on the ball size and the input, we visualized the ball motion during milling. Representative behaviors of the balls are displayed in the Movies (Supporting Information). The balls are pushed against the interior wall of the vessel due to the strong centrifugal force. Since the rotation and revolution directions in our planetary mill were the same, the circulation motion found in tumbling mill and inverse direction-type planetary mill ${ }^{32,33}$ did not occur in the simulation here. As a result, the collisions of balls are limited to a relatively small region in the packing layers pushed against the wall. The top-view snapshots of 
(a)

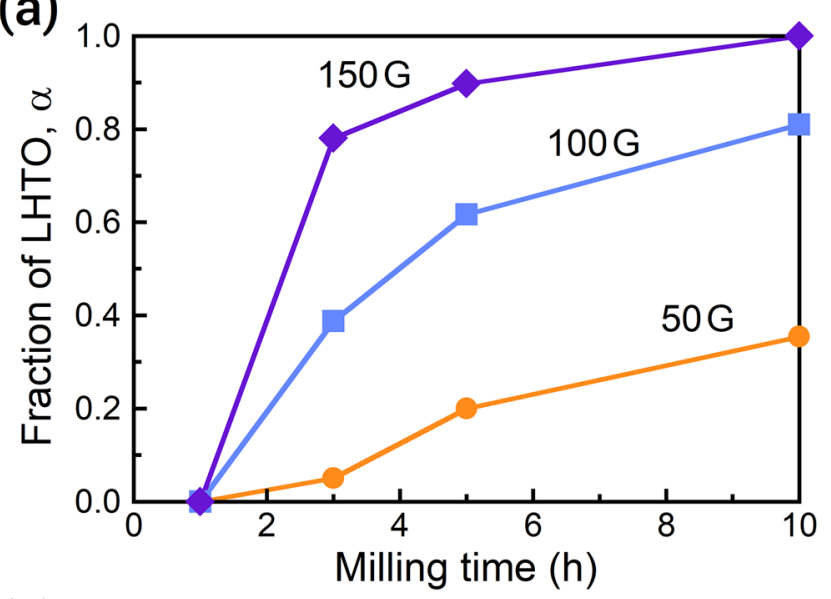

(c)

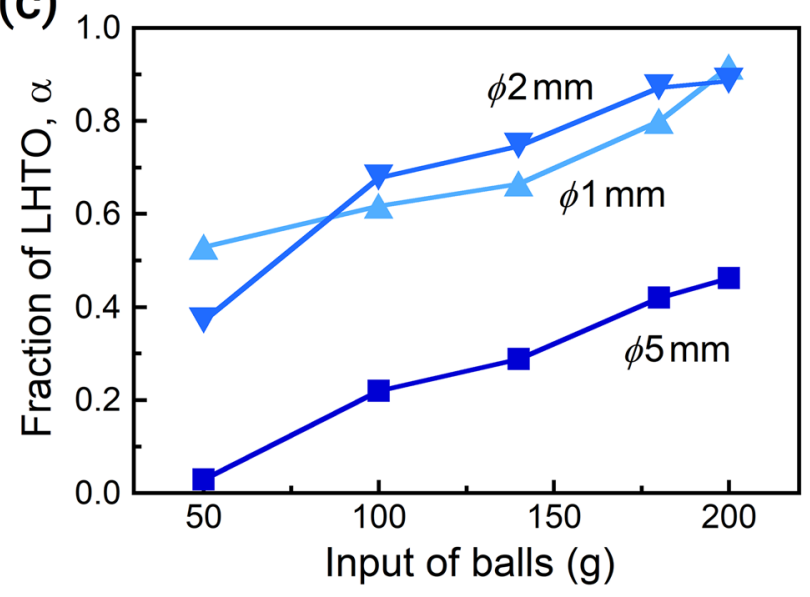

(b)

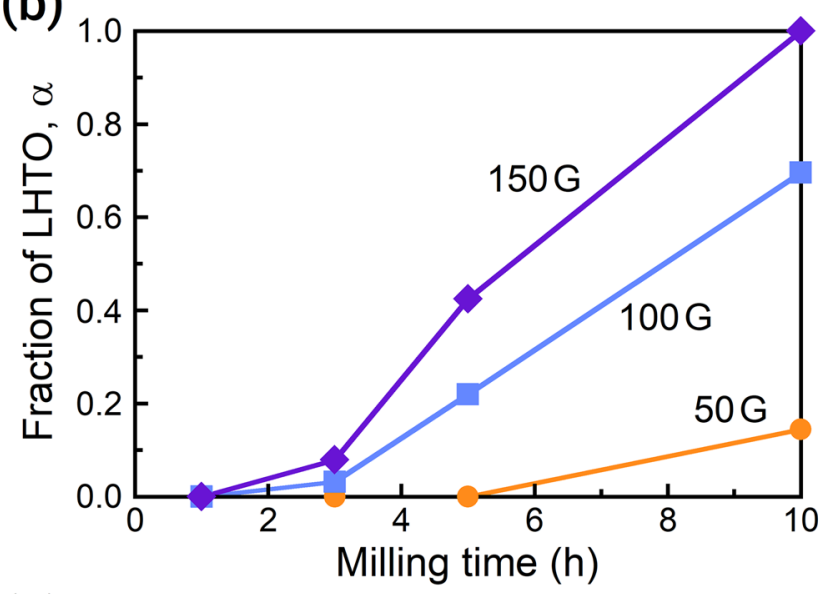

(d)

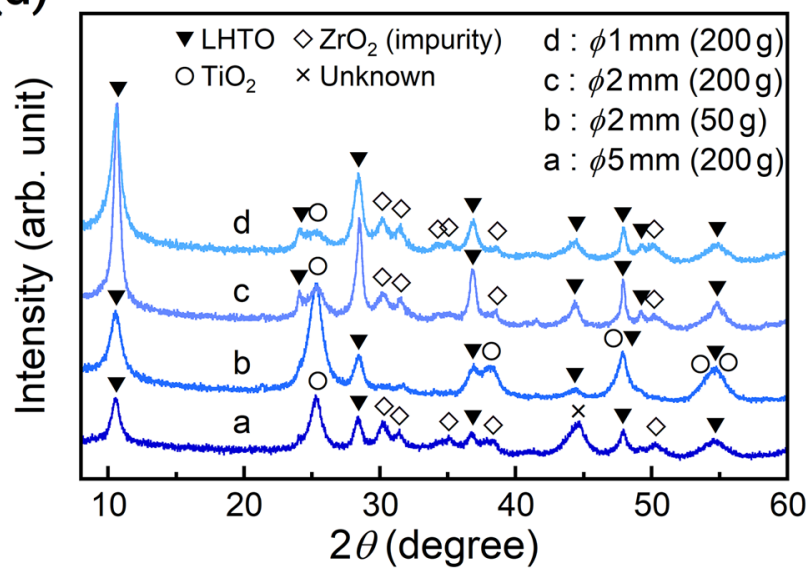

Figure 1. Formation of LHTO through the wet mechanochemical reaction. Formation fraction of LHTO after wet planetary ball milling at 50-150 G for $1-10 \mathrm{~h}$ with $100 \mathrm{~g}$ of balls at (a) $\phi 1 \mathrm{~mm}$ and (b) $\phi 5 \mathrm{~mm}$. (c) Effect of ball input and size on the reaction at $100 \mathrm{G}$ for $5 \mathrm{~h}$. (d) XRD patterns of typical products obtained at $100 \mathrm{G}$ for $5 \mathrm{~h}$ using balls of different sizes and inputs.

the interior of the vessel reveal the different packing states depending on the ball size (Fig. 3). The loading of $50 \mathrm{~g} \phi 3 \mathrm{~mm}$ balls leads to the formation of stacked packing layers, whereas the $\phi 5 \mathrm{~mm}$ or $\phi 8 \mathrm{~mm}$ balls form a single layer (Fig. 3a-c). For free balls in the packing layer, their velocity is slower than the confined ones within the layer (Fig. 3a, Movie S1). Thus, the free balls do not follow the rotation speed of the vessel. When the filling angle $(\theta)$ of balls from the vessel center exceeds $110-130^{\circ}$, the packing layers become stacked, and free-moving balls appear in the top layer. Upon loading $80 \mathrm{~g} \phi 8 \mathrm{~mm}$ balls, which is still below $\theta=100^{\circ}$, a sliding motion is permitted in both the longitudinal and lateral directions perpendicular to the centrifugal force (Fig. 3f, Movie S2). A higher ball input results in a velocity distribution in the packing layer, decreasing from the wall to the center of the vessel (Fig. 3g-i). The collision between balls in a shearing direction (tangential direction) is dominant within the layers near the wall. Meanwhile, on the packing layers, the frequency of collisions in the normal direction increases due to an increase of free-moving balls. Thus, the collision direction and frequency change with the ball input in response to the packing state of the balls.

Next, we divide the total impact energy into the normal and tangential components. The tangential component has a higher impact energy per collision, while the normal one exhibits a wide distribution in the collision frequency (Fig. 2c). Most of the impact energy comes from the tangential direction, especially at a lower ball input (Fig. 2d). According to the predicted packing states, collisions in the tangential direction occur dominantly under the conditions where the sliding motion arises with a lower ball input. In contrast, the contribution of the normal component to the total impact energy increases from 7 to $36 \%$ with an increase in the input from 50 to $260 \mathrm{~g}$ for $\phi 5 \mathrm{~mm}$ balls. The impact energy of the tangential component decreases when such collisions are constrained by surrounding balls in the stacked packing layers. With increasing ball input, since collisions in the normal direction increase due to the appearance of free-moving balls on the layer, the corresponding impact energy rises linearly. The collision frequency of the normal component is higher with smaller balls than with large ones (Fig. S2). The effect of collision frequency on the total impact energy appears at a very high ball input (Fig. 2d).

Given the cumulative impact energies for each collision direction ( $E_{n} t$ and $E_{t} t$ in normal and tangential components, respectively) based on the experimental conditions, key factors are revealed that influence the formation of LHTO in the wet mechanochemical reaction. We collected the formation ratios of LHTO obtained 
(a)

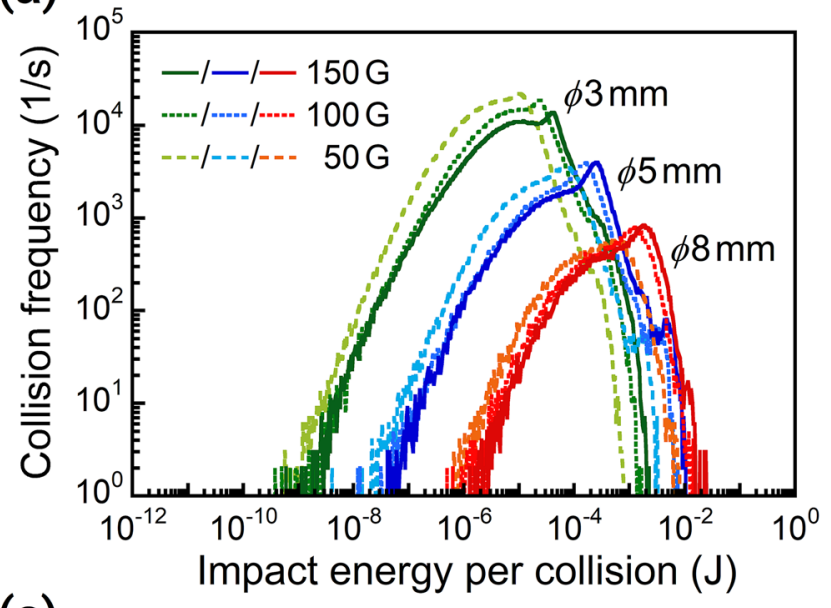

(c)

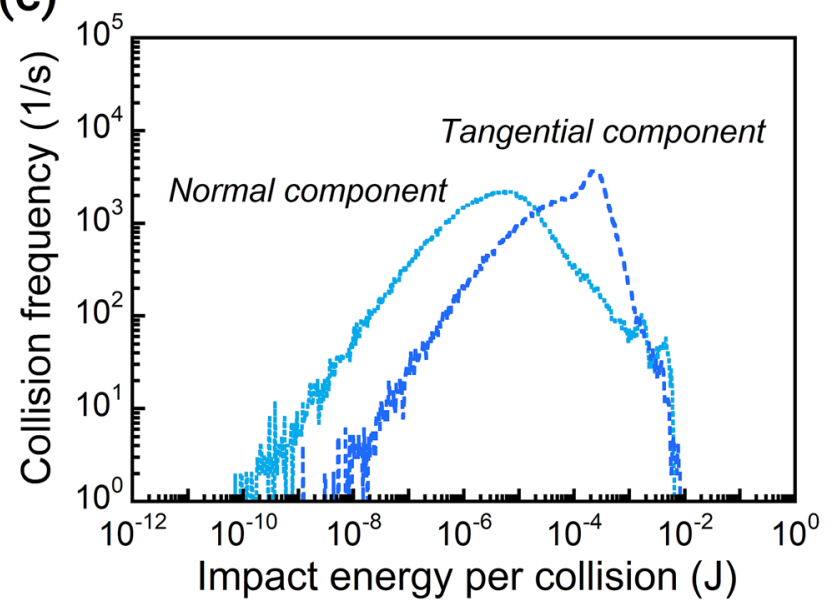

(b)

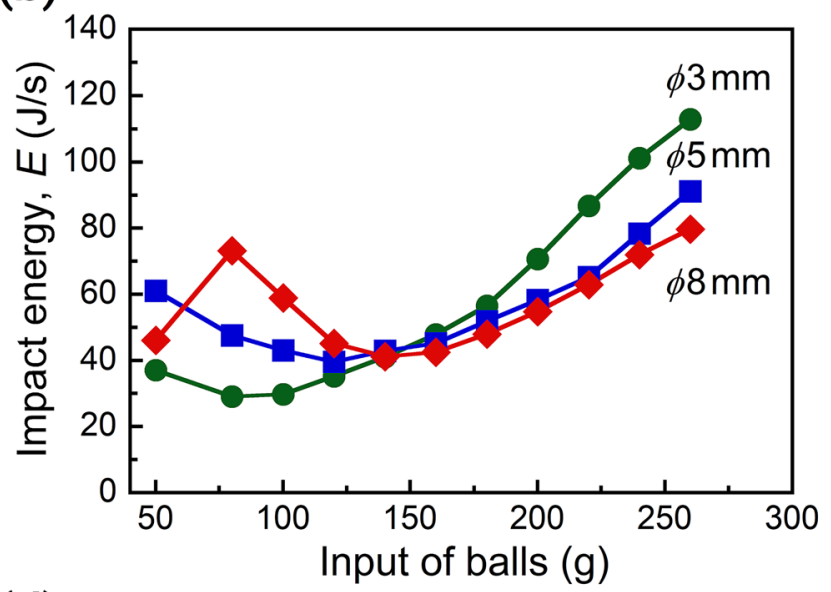

(d)

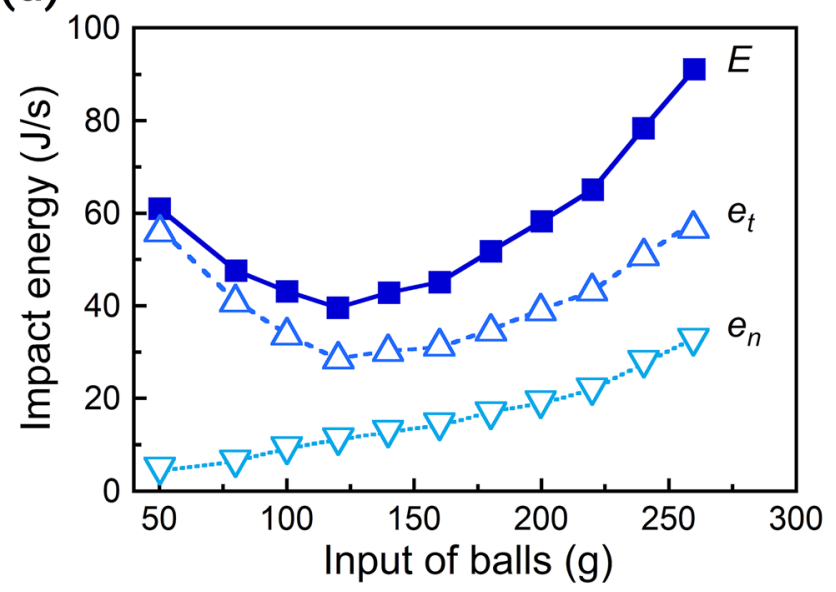

Figure 2. Impact energies between balls calculated for various planetary ball milling conditions in water. (a) Distributions of impact energy per collision at the ball input of $100 \mathrm{~g}$. (b) Relationship between the input of balls $(\phi 3-8 \mathrm{~mm})$ and the total impact energy at $150 \mathrm{G}$. (c) Distributions of impact energies in the normal and tangential directions at $150 \mathrm{G}$ with $\phi 5 \mathrm{~mm}$ balls (100 g). (d) Relationship between the input of $\phi 5 \mathrm{~mm}$ balls and the impact energies of each component at $150 \mathrm{G}$.

by varying the centrifugal acceleration, treatment time, and input for $\phi 3 \mathrm{~mm}, \phi 5 \mathrm{~mm}$, and $\phi 8 \mathrm{~mm}$ balls because the minimum ball size that can be calculated by the developed simulation software is $\phi 3 \mathrm{~mm}$. Impact energies were calculated from simulations under the treatment conditions of the experimental data used. Furthermore, the impact energy under each condition was assumed to be per $\mathrm{TiO}_{2}$ input and the cumulative value of the treatment time. The fitting results demonstrate a good correlation between the formation fraction of LHTO and the cumulative impact energy of the normal component $\left(E_{n} t\right)$ at each ball size (Fig. 4), while there are no correlations with the impact energy of the tangential component $\left(E_{t} t\right)$ or the total impact energy (Fig. S3). Thus, we suggest that ball collisions in the normal direction strongly contribute to the formation of LHTO. The use of smaller balls promotes efficient formation of LHTO at lower $E_{n} t$ values. When fixing the conditions at $E_{n} t \approx 60 \mathrm{~kJ} / \mathrm{g}$ for $\phi 3 \mathrm{~mm}$ and $\phi 5 \mathrm{~mm}$ balls, the distribution of $e_{n}$ per collision for $\phi 5 \mathrm{~mm}$ balls nearly falls within that at $\phi 3 \mathrm{~mm}$ (Fig. S4). Increasing the collision frequency in the normal direction promotes the formation of LHTO more efficiently. Unfortunately, LHTO formation could not be clearly observed when using $\phi 8 \mathrm{~mm}$ balls, although a tiny hump in the XRD pattern is attributable to it at the condition of $E_{n} t \approx 180 \mathrm{~kJ} / \mathrm{g}$ (Fig. S5). From these results, we can conclude that increasing the collision frequency in the normal direction is more effective for product formation in the wet mechanochemical reaction, as compared to merely increasing the total impact energy of collisions. The reason why collisions of balls in the normal direction contribute to the wet mechanochemical reaction is not yet fully understood but is likely associated with different pressure-changes and volumes of solution/reactants caught between balls. The motion of balls in a shearing direction (tangential direction) generates a fluid flow, which seems to disperse the collision pressure and reactants. However, a more detailed analysis is needed in the future.

The data obtained with $\phi 3 \mathrm{~mm}$ and $\phi 5 \mathrm{~mm}$ balls follow the S-shaped reaction kinetics that is known as the Gompertz model ${ }^{34}$ :

$$
\alpha=A \mathrm{e}^{\left(-\mathrm{e}^{-k\left(E_{n} t-\tau\right)}\right)}
$$




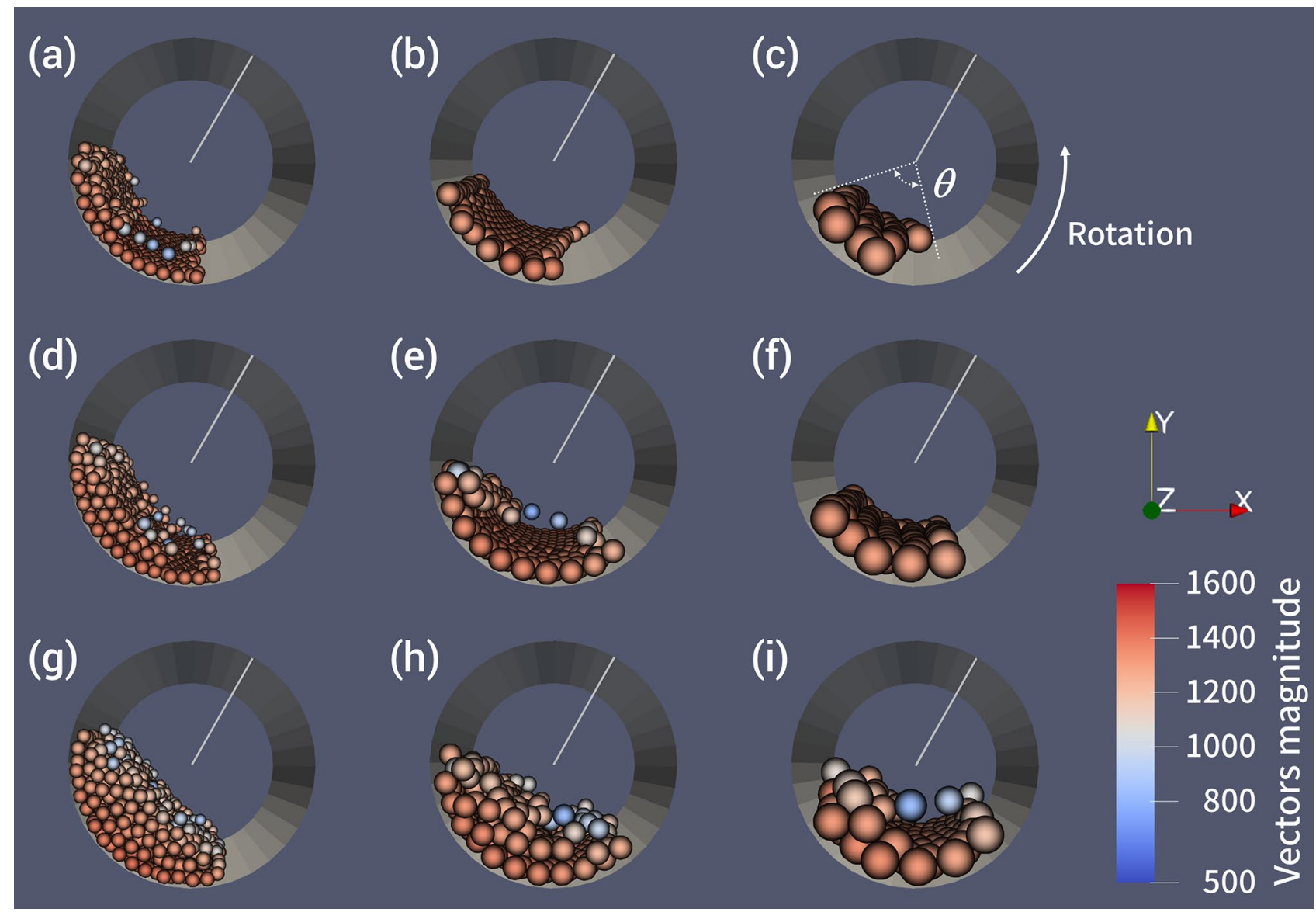

Figure 3. Snapshots of ball motion during the wet planetary ball milling at $150 \mathrm{G}$. Left column, $\phi 3 \mathrm{~mm}$; middle, $\phi 5 \mathrm{~mm}$; right, $\phi 8 \mathrm{~mm}$. Ball input: (a-c) $50 \mathrm{~g},(\mathbf{d}-\mathbf{f}) 80 \mathrm{~g}$, and $(\mathbf{g}-\mathbf{i}) 140 \mathrm{~g}$. The line from the center of the vessel indicates the direction of the center of revolution. Water is omitted from the visualization. Visualization of the simulated data was performed using ParaView software (ver. 5.8.0).

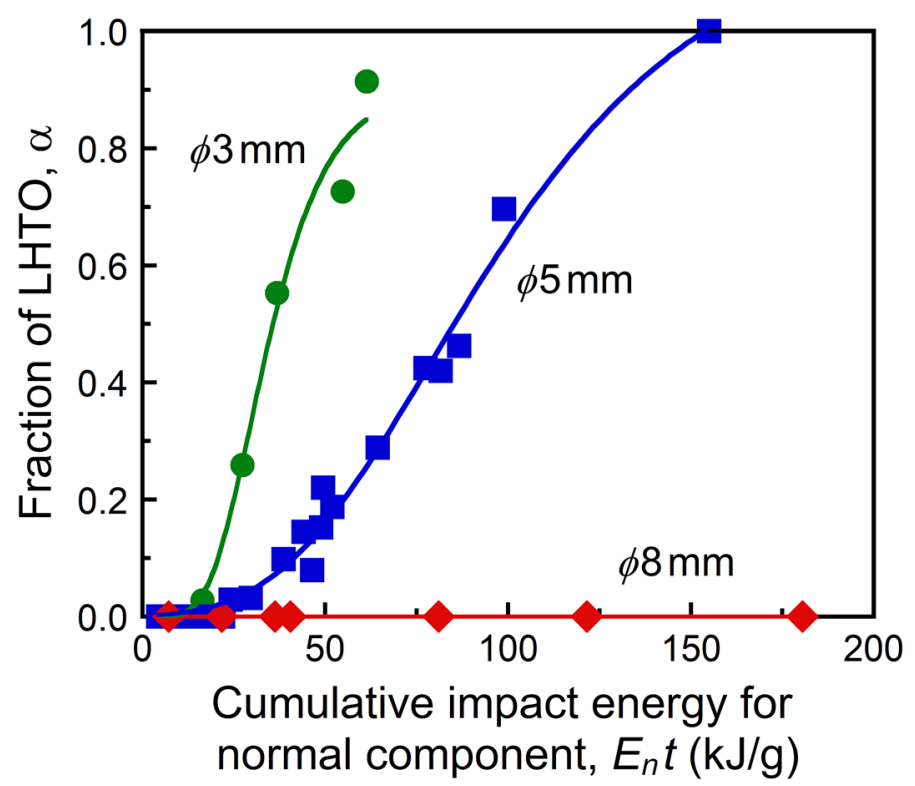

Figure 4. Effect of $E_{n} t$ on the formation fraction of LHTO through wet mechanochemical reaction. 
where $A$ is the asymptotic formation fraction, $k$ is a reaction rate constant, and $\tau$ is the $E_{n} t$ origin. In general, such sigmoidal ( $\mathrm{S}$-shaped) dependence on the energy can be described by different models: the monomolecular, the autocatalytic, and the Gompertz models ${ }^{34,35}$. The curves of both the autocatalytic and the Gompertz models contain inflection points. Further, the autocatalytic and Gompertz curves are symmetric and asymmetric with regard to the inflection point, respectively. The Gompertz model provides a good fit to our data. An example of the Gompertz model is the growth of carbon nanotubes $(\mathrm{CNTs})^{36}$. Carbon nuclei self-organized on a catalyst grow upward by thermal chemical vapor deposition and then form the $\mathrm{CNTs}^{36-38}$. A decelerating growth rate of CNTs could be due to catalyst deactivation, such as poisoning, overcoating, or evaporation. Similar to the growth model of CNTs, in the wet mechanochemical reaction, the formation of LHTO is driven by the impact energy of balls (especially in the normal direction), and the growth of LHTO driven by ion diffusion in the solution. The initial incubation/induction stage up to $E_{n} t \approx 20 \mathrm{~kJ} / \mathrm{g}$ is attributed to the step of $\mathrm{TiO}_{2}$ dissolution in $\mathrm{LiOH}$ solution. However, this step is difficult with $\phi 8 \mathrm{~mm}$ balls. When the value of $\alpha$ is higher, its growth rate decreases due to certain bottlenecks such as a lower supersaturation, long-distance diffusion, or inhibition by impurities ${ }^{39-41}$.

Finally, although the rate of the wet mechanochemical reaction depends on collision in the normal direction, the impact energy is always higher in the tangential direction (Fig. 2c,d). The distribution of impact energy in the normal direction per collision mostly overlaps with that in the tangential direction (Fig. S2). However, there two energy components differ more at higher impact energies. Such excess impact energy should lead to wear in the balls and generate frictional heat. In comparison, mechanochemical reactions under dry conditions produce less impurity from the contact between balls because of the mediating or surface coating of powder reactants ${ }^{42-44}$. As explained in Eq. (1), an increased collision frequency of ball media in solution poses a problem from wearing. Possible ways to reduce impurities in wet mechanochemical reactions include: searching for milder milling conditions $^{16}$, developing ball media that are more resistant to wearing ${ }^{45,46}$, or design the operating conditions or grinding mill to enhance collisions in the normal direction. When the collisions occur preferentially in the normal direction, one may achieve a high synthesis efficiency and suppression of impurities at the same time.

\section{Conclusion}

In summary, the formation rate of LHTO through the wet mechanochemical reaction using a planetary ball mill is mainly controlled by collision of ball media in the normal direction. The collision of balls within the solution induces nucleation and crystal growth, and the use of smaller balls at high loading accelerates the product formation. Based on simulated ball motion during milling, we conclude that the formation of LHTO is not controlled by the tangential component, even though that component accounts for more of the total impact energy per collision. Rather, LHTO formation is mainly influenced by the normal component with a wide distribution of collision frequency. A remaining problem for the wet mechanochemical reaction is impurity generation due to the wear of balls, which may be due to the differential impact energy between the tangential and normal components. Future research on the milling conditions and/or the development of new grinding mills could improve the wet mechanochemical reactions by enhancing collisions in the normal direction, in order to effectively synthesize thermodynamically unstable materials at room temperature.

\section{Methodology}

Materials and synthesis of LHTO. $\mathrm{LiOH} \cdot \mathrm{H}_{2} \mathrm{O}$ (Kanto Chemical Co., Inc., Japan) and $\mathrm{TiO}_{2}$ (anatase, ST-01, Ishihara Sangyo Kaisha, Ltd., Japan) were used as starting materials. Their mixture (3 g, Li/Ti atomic ratio $=0.905)$ and pure water $(30 \mathrm{~mL})$ were placed in a stainless-steel vessel $\left(170 \mathrm{~cm}^{3}\right.$, inner diameter $\left.=50 \mathrm{~mm}\right)$ containing $\mathrm{Y}_{2} \mathrm{O}_{3}$-stabilized $\mathrm{ZrO}_{2}$ balls (diameter, $\phi: 1-8 \mathrm{~mm}$; input: 50-200 g; Nikkato Corp., Japan). The vessel was sealed and then processed in a planetary ball mill (High-G BX254E, Kurimoto Ltd., Japan). The milling was conducted at room temperature under centrifugal acceleration of 50,100, and $150 \mathrm{G}$ by controlling the revolution speed. The ratio of rotation/revolution speeds was fixed to 0.497 . After milling for $1-10 \mathrm{~h}$, the solid product was collected via centrifugation, washed several times with water, and dried in an oven at $100{ }^{\circ} \mathrm{C}$.

Characterization. Powder XRD patterns were collected on an X-ray diffractometer (D2 PHASER, Bruker AXS, Germany) using $\mathrm{Cu} \mathrm{Ka}$ radiation to identify the crystalline phase, with steps of $0.02^{\circ}(2 \theta)$ and a counting time of $1 \mathrm{~s} / \mathrm{step}$. The fraction of LHTO formation was calculated from the intensity ratio between the strongest XRD peaks for $\mathrm{LHTO}$ and $\mathrm{TiO}_{2}$ (assuming no $\mathrm{ZrO}_{2}$ ) as follows:

$$
\alpha=\left[I_{(200)}^{\mathrm{LHTO}} /\left(I_{(200)}^{\mathrm{LHTO}}+I_{(101)}^{\mathrm{TiO}}\right)\right]
$$

Simulation. We have developed a simulation software (KIK DEM, ver.1.1) to track the motion of ball media in a wet ball mill by DEM. The motion of spherical particle $i$ (radius $r$ ) is determined by the force from the $k_{i}$ particles it is in contact with. The equations of motion for translation and rotation are as follows:

$$
\begin{gathered}
m_{i} \frac{\mathrm{d} \boldsymbol{u}_{i}}{\mathrm{~d} t}=\sum_{j=1}^{k_{i}}\left(\boldsymbol{F}_{i j}^{n}+\boldsymbol{F}_{i j}^{t}\right)+\boldsymbol{F}_{\mathrm{f}}+\boldsymbol{F}_{\mathrm{B}}+m_{i} \boldsymbol{g} \\
I_{i} \frac{\mathrm{d} \boldsymbol{\omega}_{i}}{\mathrm{~d} t}=\sum_{j=1}^{k_{i}}\left(r_{i} \boldsymbol{F}_{i j}^{t}\right)+\boldsymbol{R}_{\mathrm{r}}
\end{gathered}
$$


where $m$ is the mass of a ball, $t$ the time, $\boldsymbol{u}$ the translational velocity, $k$ the number of particles, $\boldsymbol{F}^{n}$ the normal force, $\boldsymbol{F}^{t}$ the tangential force, $\boldsymbol{F}_{\mathrm{f}}$ the fluid resistance force, $\boldsymbol{F}_{\mathrm{B}}$ the buoyant force, $\boldsymbol{g}$ the gravitational acceleration, $I$ the moment of inertia, $\boldsymbol{\omega}$ the rotational velocity, and $\boldsymbol{R}_{\mathrm{r}}$ the rolling resistance. The parameters $\boldsymbol{F}_{i j}^{n}, \boldsymbol{F}_{i j}^{t}$, and $\boldsymbol{R}_{\mathrm{r}}$ are described in detail in ref. 24 . The buoyant force was given by the volume of the ball media and the density of the fluid, while the fluid resistance force was given by the experimentally obtained equation acting on a single ball ${ }^{25}$. These interactions with fluids were considered through simplified modeling. When calculation the cumulative impact energies per unit mass under each experimental condition, we used only the mass of $\mathrm{TiO}_{2}$ because $\mathrm{LiOH}$ is soluble. Visualization of the simulated data was performed using ParaView software (ver. 5.8.0, Kitware Inc., USA, available: https://www.paraview.org).

\section{Data availability}

The authors declare that relevant data are within the manuscript.

Received: 9 November 2020; Accepted: 21 December 2020

Published online: 08 January 2021

\section{References}

1. Fischer, F., Fendel, N., Greiser, S., Rademann, K. \& Emmerling, F. Impact is important-systematic investigation of the influence of milling balls in mechanochemical reactions. Org. Process Res. Dev. 21, 655-659 (2017).

2. Howard, J. L., Cao, Q. \& Browne, D. L. Mechanochemistry as an emerging tool for molecular synthesis: what can it offer?. Chem. Sci. 9, 3080-3094 (2018).

3. Ahiavi, E. et al. Mechanochemical synthesis and ion transport properties of $\mathrm{Na}_{3} \mathrm{OX}\left(\mathrm{X}=\mathrm{Cl}, \mathrm{Br}, \mathrm{I}\right.$ and $\left.\mathrm{BH}_{4}\right)$ antiperovskite solid electrolytes. J. Power Sources 471, 228489 (2020).

4. Maurice, D. R. \& Coutney, T. H. The physics of mechanical alloying: a first report. Metall. Trans. A 21, 289-303 (1990).

5. Suryanarayana, C. Mechanical alloying and milling. Prog. Mater. Sci. 46, 1-184 (2001).

6. Humphry-Baker, S. A., Garroni, S., Delogu, F. \& Schuh, C. A. Melt-driven mechanochemical phase transformations in moderately exothermic powder mixtures. Nat. Mater. 15, 1280-1286 (2016).

7. Boulineau, S., Courty, M., Tarascon, J.-M. \& Viallet, V. Mechanochemical synthesis of Li-argyrodite $\mathrm{Li}_{6} \mathrm{PS}_{5} X(X=\mathrm{Cl}, \mathrm{Br}, \mathrm{I})$ as sulfur-based solid electrolytes for all solid state batteries application. Solid State Ion. 221, 1-5 (2012).

8. Huot, J. et al. Mechanochemical synthesis of hydrogen storage materials. Prog. Mater. Sci. 58, 30-75 (2013)

9. Šepelák, V., Bégin-Colin, S. \& Le Caër, G. Transformations in oxides induced by high-energy ball-milling. Dalton Trans. 41, $11927-11948$ (2012).

10. Šepelák, V., Düvel, A., Wilkening, M., Becker, K.-D. \& Heitjans, P. Mechanochemical reactions and syntheses of oxides. Chem. Soc. Rev. 42, 7507-7520 (2013).

11. Fecht, H.-J. Nanostructure formation by mechanical attrition. NanoStruct. Mater. 6, 33-42 (1995).

12. Kozawa, T. \& Naito, M. Mechanically induced formation of metastable $\chi$ - and $\kappa$ - $\mathrm{Al}_{2} \mathrm{O}_{3}$ from boehmite. Adv. Powder Technol. 27, 935-939 (2016).

13. Kosova, N. V., Khabibullin, A. K. \& Boldyrev, V. V. Hydrothermal reactions under mechanochemical treating. Solid State Ionics 101-103, 53-58 (1997).

14. Shuk, P. et al. Mechanochemical-hydrothermal preparation of crystalline hydroxyapatite powders at room temperature. J. Mater. Res. 16, 1231-1234 (2001).

15. Suchanek, W. L. et al. Mechanochemical-hydrothermal synthesis of carbonated apatite powders at room temperature. Biomaterials 23, 699-710 (2002).

16. Kozawa, T., Suzuki, Y. \& Naito, M. Scalable synthesis of $\mathrm{Sr}_{3} \mathrm{Al}_{2}(\mathrm{OH})_{12}$ hydrogarnet by wet milling and its thermal decomposition behavior. Mater. Chem. Phys. 212, 245-251 (2018).

17. Suzuki, S., Kozawa, T., Murakami, T. \& Naito, M. Mechanochemical-hydrothermal synthesis of layered lithium titanate hydrate nanotubes at room temperature and their conversion to $\mathrm{Li}_{4} \mathrm{Ti}_{5} \mathrm{O}_{12}$. Mater. Res. Bull. 90, 218-223 (2017).

18. Kozawa, T., Fukuyama, K., Kondo, A. \& Naito, M. Wet mechanical route to synthesize morphology-controlled $\mathrm{NH}_{4} \mathrm{MnPO}_{4} \cdot \mathrm{H}_{2} \mathrm{O}$ and its conversion reaction into $\mathrm{LiMnPO}_{4}$. ACS Omega 4, 5690-5695 (2019).

19. Baláž, P. et al. Hallmarks of mechanochemistry: from nanoparticles to technology. Chem. Soc. Rev. 42, 7571-7637 (2013).

20. Dachille, F. \& Roy, R. High-pressure transformations in laboratory mechanical mixers and mortars. Nature 186, 34-71 (1960).

21. Boldyrev, V. V. Hydrothermal reactions under mechanochemical action. Powder Technol. 122, 247-254 (2002).

22. Delogu, F. \& Cocco, G. Weakness of the "hot spots" approach to the kinetics of mechanically induced phase transformations. J. Alloys Compd. 465, 540-546 (2008).

23. Fuerstenau, D. W. \& Abouzeid, A.-Z.M. The energy efficiency of ball milling in comminution. Int. J. Miner. Process. 67, 161-185 (2002).

24. Watanabe, R., Kushimoto, K., Ishihara, S. \& Kano, J. Determination method of coefficients of particle and rolling friction in distinct element method. J. Soc. Powder Technol. Jpn. 56, 218-225 (2019) ((in Japanese)).

25. Mori, H., Mio, H., Kano, J. \& Saito, F. Ball mill simulation in wet grinding using a tumbling mill and its correlation to grinding rate. Powder Technol. 143-144, 230-239 (2004).

26. $\mathrm{Li}$, N. et al. Hydrothermal synthesis of layered $\mathrm{Li}_{1.81} \mathrm{H}_{0.19} \mathrm{Ti}_{2} \mathrm{O}_{5} \cdot \mathrm{xH}_{2} \mathrm{O}$ nanosheets and their transformation to single-crystalline $\mathrm{Li}_{4} \mathrm{Ti}_{5} \mathrm{O}_{12}$ nanosheets as the anode materials for Li-ion batteries. CrystEngComm 14, 6435-6440 (2012).

27. Sha, Y., Zhao, B., Ran, R., Cai, R. \& Shao, Z. Synthesis of well-crystallized $\mathrm{Li}_{4} \mathrm{Ti}_{5} \mathrm{O}_{12}$ nanoplates for lithium-ion batteries with outstanding rate capability and cycling stability. J. Mater. Chem. A 1, 13233-13243 (2013).

28. Xiao, L. et al. Facile synthesis of $\mathrm{Li}_{4} \mathrm{Ti}_{5} \mathrm{O}_{12}$ nanosheets stacked by ultrathin nanoflakes for high performance lithium ion batteries. J. Mater. Chem. A 1, 14618-14626 (2013).

29. Xu, R., Li, J., Tan, A., Tang, Z. \& Zhang, Z. Novel lithium titanate hydrate nanotubes with outstanding rate capabilities and long cycle life. J. Power Sources 196, 2283-2288 (2011).

30. Sugita, M., Tsuji, M. \& Abe, M. Synthetic inorganic ion-exchange materials. LVIII. Hydrothermal synthesis of a new layered lithium titanate and its alkali ion exchange. Bull. Chem. Soc. Jpn. 63, 1978-1984 (1990).

31. Yang, J., Jin, Z., Wang, X., Li, W., Zhang, J., Zhang, S., Guo, X. \& Zhang, Z. Study on composition, structure and formation process of nanotube $\mathrm{Na}_{2} \mathrm{Ti}_{2} \mathrm{O}_{4}(\mathrm{OH})_{2}$. Dalton Trans. 3898-3901 (2003).

32. Hashimoto, H., Lee, G. G. \& Watanabe, R. Simulation of ball motion for analysis of coating phenomena during tumbler-ball milling of Cu powder. Mater. Trans. JIM 35, 40-45 (1994).

33. Watanabe, R., Hashimoto, H. \& Lee, G. G. Computer simulation of milling ball motion in mechanical alloying (overview). Mater. Trans. JIM 36, 102-109 (1995).

34. Winsor, C. P. The Gompertz curve as a growth curve. Proc. Natl. Acad. Sci. USA 18, 1-8 (1932). 
35. Richards, F. J. A flexible growth function for empirical use. J. Exp. Bot. 10, 290-300 (1959).

36. Bedewy, M., Meshot, E. R., Reinker, M. J. \& Hart, A. J. Population growth dynamics of carbon nanotubes. ACS Nano 5, 8974-8989 (2011).

37. Futaba, D. N. et al. Kinetics of water-assisted single-walled carbon nanotube synthesis revealed by a time-evolution analysis. Phys. Rev. Lett. 95, 056104 (2005).

38. Bedewy, M. et al. Collective mechanism for the evolution and self-termination of vertically aligned carbon nanotube growth. J. Phys. Chem. C 113, 20576-20582 (2009).

39. Vekilov, P. G. What determines the rate of growth of crystals from solution?. Cryst. Growth Des. 7, 2796-2810 (2007).

40. Lin, H.-X. et al. Supersaturation-dependent surface structure evolution: from ionic, molecular to metallic micro/nanocrystals. J. Am. Chem. Soc. 135, 9311-9314 (2013).

41. van Enckevort, W. J. P., van der Berg, A. C. J. F., Kreuwel, K. B. G., Derksen, A. J. \& Couto, M. S. Impurity blocking of growth steps: experiments and theory. J. Cryst. Growth 166, 156-161 (1996).

42. Morimoto, H., Yamashita, H., Tatsumisago, M. \& Minami, T. Mechanochemical synthesis of new amorphous materials of $60 \mathrm{Li}_{2} \mathrm{~S} \cdot 40 \mathrm{SiS}_{2}$ with high lithium ion conductivity. J. Am. Ceram. Soc. 82, 1352-1354 (1999).

43. Stojanovic, B. D. et al. Mechanochemical synthesis of barium titanate. J. Eur. Ceramic. Soc. 25, 1985-1989 (2005).

44. Mancheva, M. N., Iordanova, R. S., Klissurski, D. G., Tyuliev, G. T. \& Kunev, B. N. Direct mechanochemical synthesis of nanocrystalline $\mathrm{NiWO}_{4}$. J. Phys. Chem. C 111, 1101-1104 (2007).

45. Lee, W.-H. et al. $\mathrm{Al}_{2} \mathrm{O}_{3}$-nanodiamond composite coatings with high durability and hydrophobicity prepared by aerosol deposition. Surf. Coat. Technol. 206, 4679-4684 (2012).

46. Greco, A., Mistry, K., Sista, V., Eryilmaz, O. \& Erdemir, A. Friction and wear behaviour of boron based surface treatment and nano-particle lubricant additives for wind turbine gearbox applications. Wear 271, 1754-1760 (2011).

\section{Acknowledgments}

This work was supported by the High Performance of Ceramics and Manufacturing Process, Adaptable and Seamless Technology Transfer Program (A-STEP) through target-driven R\&D from the Japan Science Technology Agency (JST) (Grant Number JPMJTS1615), and by the JWRI Young Researcher Grant Program.

\section{Author contributions}

T.K. conducted the simulation, organized the experimental and simulated data, drew figures. K.F. collected the experimental data. K.K., S.I., and J.K. developed the simulation software. A.K. and M.N. supported the data analysis of the simulation. The paper was written by T.K. All authors reviewed the manuscript.

\section{Competing interests}

The authors declare no competing interests.

\section{Additional information}

Supplementary information is available for this paper at https://doi.org/10.1038/s41598-020-80342-w.

Correspondence and requests for materials should be addressed to T.K.

Reprints and permissions information is available at www.nature.com/reprints.

Publisher's note Springer Nature remains neutral with regard to jurisdictional claims in published maps and institutional affiliations.

Open Access This article is licensed under a Creative Commons Attribution 4.0 International License, which permits use, sharing, adaptation, distribution and reproduction in any medium or format, as long as you give appropriate credit to the original author(s) and the source, provide a link to the Creative Commons licence, and indicate if changes were made. The images or other third party material in this article are included in the article's Creative Commons licence, unless indicated otherwise in a credit line to the material. If material is not included in the article's Creative Commons licence and your intended use is not permitted by statutory regulation or exceeds the permitted use, you will need to obtain permission directly from the copyright holder. To view a copy of this licence, visit http://creativecommons.org/licenses/by/4.0/.

(C) The Author(s) 2021 